\title{
Corrigendum
}

\section{Corrigendum to: Protective effects of lutein against vancomycin- induced acute renal injury in mice via upregulation of peroxisome proliferator-activated receptor gamma/nuclear factor erythroid 2-related factor 2 and inhibition of nuclear factor-kappaB/ caspase 3}

Promise M. Emeka ${ }^{1, *}$, Sahibzada T. Rasool ${ }^{2}$, Mohamed A. Morsy ${ }^{1,3}$, Mohamed I. Hairul Islam ${ }^{4}$, and Muhammad S. Chohan ${ }^{2}$

Departments of ${ }^{1}$ Pharmaceutical Sciences and ${ }^{2}$ Biomedical Sciences, College of Clinical Pharmacy, King Faisal University, Al-Ahsa 31982, Saudi Arabia, ${ }^{3}$ Department of Pharmacology, Faculty of Medicine, Minia University, El-Minia 61511, Egypt, ${ }^{4}$ Department of Biological Sciences, College of Science, King Faisal University, Al-Ahsa 31982, Saudi Arabia

\section{Corrigendum to:}

Korean J Physiol Pharmacol 2021;25(4):321-331.

Published online July 1, 2021. https://doi.org/10.4196/kjpp.2021.25.4.321

The originally published version of this article contained an error in the title. The author apologizes for any inconvenience that it may have caused.

Before correction: Protective effects of lutein against vancomycin-induced acute renal injury in mice via upregulation of peroxisome proliferator-activated receptor gamma/nuclear factor erythroid 2-related factor 2 and inhibition nuclear factor-kappaB/caspase 3

After correction: Protective effects of lutein against vancomycin-induced acute renal injury in mice via upregulation of peroxisome proliferator-activated receptor gamma/nuclear factor erythroid 2-related factor 2 and inhibition of nuclear factor-kappaB/caspase 3

*Correspondence: Promise M. Emeka, E-mail: pemeka@kfu.edu.sa 